\title{
Article \\ Development and Evaluation of a Dual-Layer-Offset PET Detector Constructed with Different Reflectors
}

\author{
Xi Zhang ${ }^{1,2} \mathbb{D}$, Xin $\mathbf{Y u}^{2}$, Zhiliang Zhu ${ }^{1}$, Hongsen $\mathbf{u}^{2}{ }^{2}$, Heng Zhang ${ }^{2}$, Yibin Zhang ${ }^{1}$, Zheng Gu ${ }^{1}$, Jianfeng Xu ${ }^{2, *}$, \\ Qiyu Peng ${ }^{1, *}$ and Siwei Xie ${ }^{1, *}$
}

check for

updates

Citation: Zhang, X.; Yu, X.; Zhu, Z.;

Yu, H.; Zhang, H.; Zhang, Y.; Gu, Z.; Xu, J.; Peng, Q.; Xie, S. Development and Evaluation of a Dual-LayerOffset PET Detector Constructed with Different Reflectors. Crystals 2022, 12, 93. https://doi.org/ $10.3390 /$ cryst 12010093

Academic Editors: Ana Pilar Valerga Puerta and Hong Joo Kim

Received: 29 November 2021

Accepted: 2 January 2022

Published: 11 January 2022

Publisher's Note: MDPI stays neutral with regard to jurisdictional claims in published maps and institutional affiliations.

Copyright: (c) 2022 by the authors. Licensee MDPI, Basel, Switzerland. This article is an open access article distributed under the terms and conditions of the Creative Commons Attribution (CC BY) license (https:// creativecommons.org/licenses/by/ $4.0 /)$.
1 Institute of Biomedical Engineering, Shenzhen Bay Laboratory, Shenzhen 518132, China; xizhang@hust.edu.cn (X.Z.); zhiliang_96@163.com (Z.Z.); zhangyb@szbl.ac.cn (Y.Z.); guzheng@szbl.ac.cn (Z.G.)

2 School of Mechanical Science and Engineering, Huazhong University of Science and Technology, Wuhan 430074, China; M202070480@hust.edu.cn (X.Y.); m201970550@hust.edu.cn (H.Y.); M202170850@hust.edu.cn (H.Z.)

* Correspondence: jfxu@hust.edu.cn (J.X.); universalring@163.com (Q.P.); xiesw@szbl.ac.cn (S.X.)

\begin{abstract}
Dual-layer-offset or multi-layer-offset design of a PET detector can improve spatial resolution while maintaining high sensitivity. In this study, three dual-layer-offset LYSO detectors with three different reflectors (ESR, Toray, and $\mathrm{BaSO}_{4}$ ) were developed. The top layer consisted of a $17 \times 17$ array of crystals $1 \times 1 \times 6.5 \mathrm{~mm}^{3}$ in size and the bottom layer consisted of an $18 \times 18$ array of crystals $1 \times 1 \times 9.5 \mathrm{~mm}^{3}$ in size. Neither light guides nor optical glue were used between the two layers of crystals. A custom-designed electronics system, composed of a $6 \times 6$ SiPM array, two FPC cables, and a custom-designed data processing module, was used to read out signals. An optimized interaction-decoding algorithm using the center of gravity to determine the position and threshold of analog signals for timing methods was applied to generate decoding flood histograms. The detector performances, in terms of peak to valley ratio of the flood histograms and energy resolutions, were calculated and compared. The dual-layer-offset PET detector constructed with $\mathrm{BaSO}_{4}$ reflectors performed much better than the other two reflectors in both crystal identification and energy resolution. The average peak-to-valley ratio and the energy resolution were approximately 7 and $11 \%$, respectively. In addition, the crystals in the bottom layer showed better performance at crystal identification than those in the top layer. This study can act as a reference providing guidance in choosing scintillator reflectors for multi-layer dedicated DOI detectors designed for small-animal PET imaging.
\end{abstract}

Keywords: high resolution; PET detector; dual-layer-offset; reflectors

\section{Introduction}

Positron emission tomography (PET) is a molecular imaging modality that uses a radioactive tracer to locate and measure changes in metabolic processes for biomedical and diagnostic research [1-5]. The fundamental mechanism of PET is the detection of the gamma photon pairs produced by positron annihilation. Image reconstruction techniques can then be used to obtain the distribution of radiotracers. Single-crystal scintillators, such as lutetium-yttrium oxyorthosilicate (LYSO), bismuth germanate (BGO), and gadolinium aluminum gallium garnet (GAGG) have been studied and found to be advantageous over other materials for PET applications [6]. LYSO is widely used in state-of-the-art PET and PET detectors for its high light yield and short decay time.

There is a trade-off between the key elements of sensitivity and spatial resolution in PET systems. Moses developed the following empirical formula to evaluate the influence of related parameters on the spatial resolution of a PET detector [7]: 


$$
R_{F W H M}=\mu \sqrt{\left(\frac{d}{2}\right)^{2}+(0.0044 R)^{2}+s^{2}+b^{2}+\left(\frac{1.25 r}{\sqrt{r^{2}+R^{2}}}\right)^{2}}
$$

where $d$ is the crystal width, $R$ is the diameter of the system, $s$ is the positron range, $b$ is the error factor, and $\mu$ is a multiplicative factor obtained from the reconstruction method. Decreasing the cross-section of the crystal is one direct and valid method to improve spatial resolution. Long crystals with small cross-sections are required for high spatial resolution and high sensitivity [8]. However, long crystals can lead to mislocation of light response lines (LOR) and parallax effects, which deteriorate image quality. Choghadi et al. proposed a calculation algorithm of the radial resolution at the given point within the field of view, which can be calculated as following:

$$
\tau \approx \sqrt{\left(\frac{d}{2}\right)^{2}+\frac{w^{2}-d^{2}}{D^{2}} r^{2}}
$$

where $w$ is the depth of interaction (DOI) resolution, $d$ is the detector pitch, $D$ is the ring diameter, and $r$ is the radial distance from the center of the ring [9]. Note that the reconstruction, individual coupling, and readout were assumed to be perfect, and the positron range and angular nonlinearity were ignored. Developing such high-resolution detectors with DOI capability is of great interest for both preclinical and clinical PET applications.

Significant efforts have been invested in recent decades to develop a PET detector with DOI capability. On the one hand, dual-ended readout detector designs can achieve promising coincidence of time resolution (CTR) and DOI resolution [9-11]. For instance, Kang et al. optimized the surface processing and reflector design and achieved a sub-200 ps CTR and sub-3 mm DOI resolution [10]. Kuang et al. developed an MRI-compatible detector with all crystals resolved and the detectors achieving a DOI resolution of $1.96 \mathrm{~mm}$ and an energy resolution of $18.9 \%$ [11]. The drawback of these dual-ended readout detectors is that they are too costly to be commercialized because of the enormous number of photodetectors and other readout circuits required. The typical applications of dual-layer DOI detection modules are the GE Healthcare eXplore VISTA PET scanner and Shimadzu Clairvivo small animal PET [12,13]. On the other hand, creative single-ended readout detector designs have also been proposed, including phoswich detectors, dual-layer-offset and multilayer-offset detectors, and several methods of light sharing [14,15]. Phoswich detectors are commonly used for a wide range of applications, academic as well as industrial, including medical, security, nuclear, etc. [16-23]. For example, a phoswich detector using a two-layer crystal of LYSO and BGO was developed to reject the cross-layer Compton scatter events and improve the identification fraction [24]. A pulse shape discrimination technique utilized the ECAT high-resolution research tomography (HRRT) [25]. There are two main strategies for DOI measurement using a light-sharing method: (1) Designing light-sharing windows with reflectors of different shapes, sizes and materials such that the light distributions can be manipulated to encode DOI information [26,27]. Zhang et al. have proposed a depth-encoding PET detector using four crystals to one SiPM that could obtain approximately $3 \mathrm{~mm}$ DOI resolution and sub-200 ps coincidence timing resolution [28]. (2) Arranging a light guide at the top of the crystal arrays to guide the optical photons to specific photodetectors and thus decode the DOI. LaBella et al. designed and fabricated a novel prismatoid light-guide array that realized a DOI resolution of $2.5 \mathrm{~mm}$ and an energy resolution of 9\% $[29,30]$. Continuous crystals are also used in small animal PET scanners [31]. For example, Balcerzyk et al. coupled crystals to a finely segmented array of photomultipliers to extract DOI data, while also greatly increasing the system cost and complexity [31].

Dual-layer-offset and multi-layer-offset detectors have several advantages over the aforementioned approaches, including relatively compact readout electronics, precise DOI measurements, and practical crystal array assembly [8,32-38]. For instance, Ito et al. have conducted simulation and detector design work focused on four-layer DOI crystals of 
dimensions $1.5 \times 1.5 \times 7 \mathrm{~mm}^{3}$ with a crystal pitch of $1.565 \mathrm{~mm}[34,37]$. Wei et al. and Zhang et al. utilized dual-layer-offset detectors for PET system applications $[35,36]$. The main characteristics of detectors, especially the energy and spatial resolutions, depend strongly on the reflectors between crystals. The most common reflectors used in detector manufacturing are barium sulfate $\left(\mathrm{BaSO}_{4}\right)$, enhanced specular reflector (ESR), and Toray film (Toray Industries Inc., Tokyo, Japan) [39-41].

In this study, three dual-layer-offset LYSO detectors with the above-mentioned reflector materials were fabricated, investigated, and compared, in order to optimize the reflector configuration. An optimized interaction decoding algorithm using center of gravity (COG) and the threshold method was applied to generate the flood histograms. The peak-tovalley ratios of the decoding spot were calculated from the flood histograms to evaluate the decoding performance. The results indicated that the $\mathrm{BaSO}_{4}$ reflector performed substantially better than the other two reflectors in both crystal identification and energy resolution. This paper provides referable guidance for choosing scintillator reflectors for multi-layer dedicated DOI detectors designed for small-animal PET imaging.

\section{Methods}

\subsection{Crystal Array}

Three dual-layer-offset LYSO arrays consisting of $17 \times 17$ crystals $\left(1 \times 1 \times 6.5 \mathrm{~mm}^{3}\right)$ and $18 \times 18$ crystal $\left(1 \times 1 \times 9.5 \mathrm{~mm}^{3}\right)$, as shown in Figure 1 , were fabricated by Meishanboya Material Co., Ltd. All LYSO crystals are mechanically polished and connected with different internal reflectors. The first type, widely used in PET detectors, is the enhanced specular reflector (ESR) (3M, St. Paul, MN, USA) with a 98.5\% reflectivity. The second reflector is the Toray lumirror E60 film (Toray Industries Inc., Japan). The third reflector is a self-made mixture of $\mathrm{BaSO}_{4}$ and optical glue. The surface by which gamma photons enter, as well as the lateral surfaces, were wrapped with an aluminum foil of $0.05 \mathrm{~mm}$ thickness. To simplify the process of assembly and keep detectors unified, neither light guides nor optical glue were placed between the two layers, which may require higher electronic readout capability. A small amount of optical glue $(0.01 \mathrm{~mm}$ in thickness) was used between the two layer of crystals for connecting, which is different from reference [42]. The overall dimensions of the three crystal arrays differed slightly due to the different thicknesses of the reflectors: $(19.32 \times 19.32+20.44 \times 0.44) \times 16.20 \mathrm{~mm}^{3}$ for the ESR array, $(19.34 \times 19.34+20.46 \times 20.46) \times 16.18 \mathrm{~mm}^{3}$ for the Toray array, and $(19.28 \times 19.28+20.40$ $\times 20.40) \times 16.16 \mathrm{~mm}^{3}$ for the $\mathrm{BaSO}_{4}$ array.

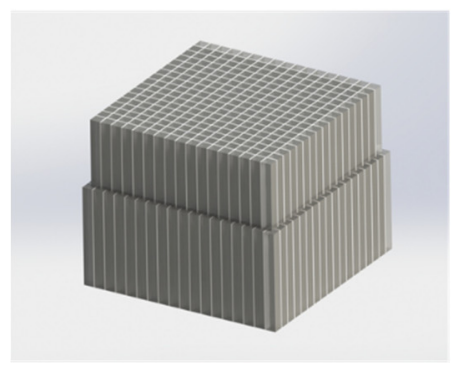

(a)

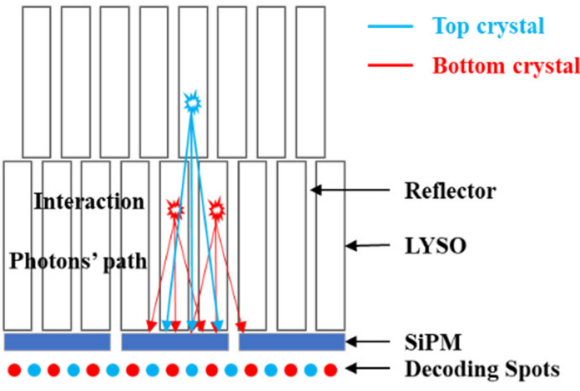

(b)

Figure 1. Cont. 


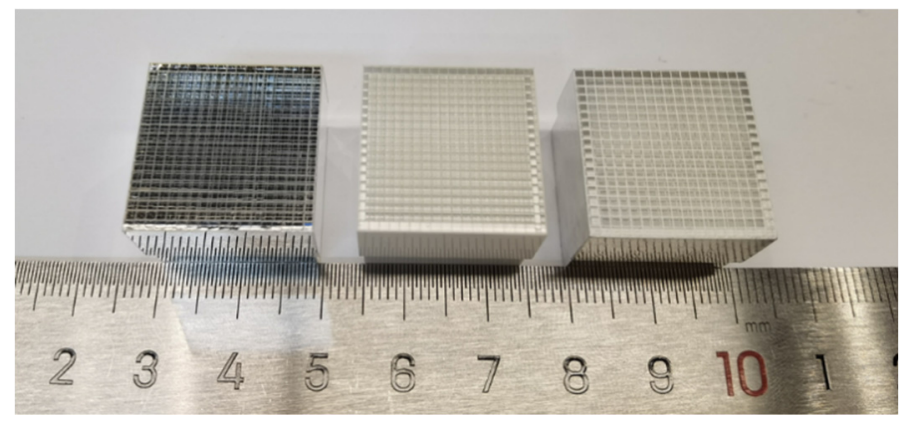

(c)

Figure 1. (a) A model of the dual-layer-offset crystal array. (b) A diagram of the relationship between the decoding spots and the crystal layers where the interactions occur. The decoding spots of the top crystals and the bottom crystals alternate. (c) A picture of the three crystal arrays constructed with three different reflectors: ESR, Toray, and $\mathrm{BaSO}_{4}$.

\subsection{Detector Measurements}

The readout electronics are composed of a $6 \times 6$ Silicon Photomultipliers (SiPM) array, two FPC cables, and a custom-designed data processing module (Figure 2). The SiPM has a $3 \times 3 \mathrm{~mm}^{2}$ active area and contains 5601 microcells, each covering an area of $35 \times 35 \mu \mathrm{m}^{2}$ (MicroFJ-30035-TSV, SensL). The bias voltage is set to $29.5 \mathrm{~V}$. The ratio of the crystal size to the SiPM size is approximately 1:3. The interval between the two SiPMs was $0.2 \mathrm{~mm}$. The overall size of the SiPM array was $19.96 \times 19.96 \mathrm{~mm}^{2}$. The SiPM array is connected to the data processing module via the FPC cables and a hub board. The core chip of the electronic module is a Cyclone 10 FPGA from Altera, which reads out all 36 channels of signals and transmits the data to a host PC. The details of the readout electronics are described in reference [43]. The readout electronics showed excellent accuracy, linearity, repeatability, and uniformity; the energy spectra were not corrected for the saturation of the SiPMs.

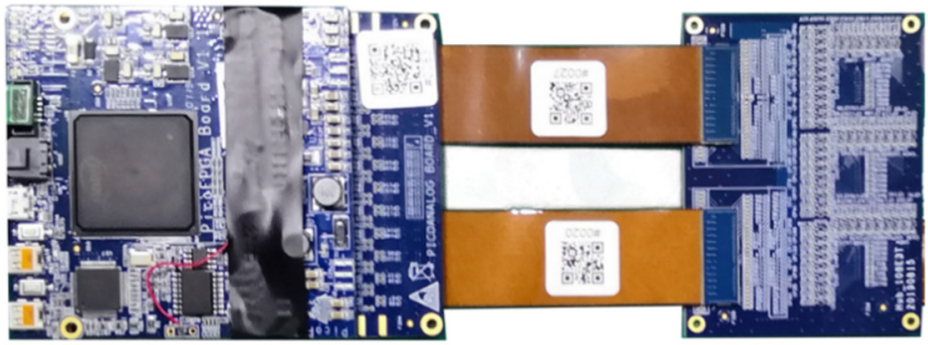

(a)

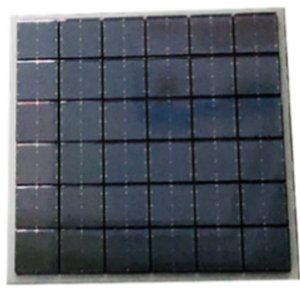

(b)

Figure 2. Pictures of (a) the custom-designed electronic system and (b) the SiPM array.

The crystal arrays were coupled to the SiPM array by air to a form a detector, with four layers of Teflon tape wrapped around it. The detectors were irradiated by a ${ }^{22} \mathrm{Na}$ point source located $5 \mathrm{~cm}$ above the top end of the crystal arrays. The radiation dose and the diameter of active area were $753 \mathrm{kBq}$ and $0.25 \mathrm{~mm}$, respectively. The data acquisition time was $250 \mathrm{~s}$. The temperature was set constant at $25^{\circ} \mathrm{C}$ during the experiments.

\subsection{Data Analysis}

The flood histogram is the most intuitive method to evaluate the decoding performance of a detector. First, an energy window of [425 keV, $625 \mathrm{keV}]$ was applied to all detected 
events in order to reject the Compton events. The interaction was then calculated using the COG method:

$$
\left\{\begin{array}{l}
x=\frac{256}{6} *\left(\frac{E_{r, 1}+2 * E_{r, 2}+3 * E_{r, 3}+4 * E_{r, 4}+5 * E_{r, 5}+6 * E_{r, 6}}{E_{t o t a l}}-1\right) \\
y=\frac{256}{6} *\left(\frac{E_{c, 1}+2 * E_{c, 2}+3 * E_{c, 3}+4 * E_{c, 4}+5 * E_{c, 5}+6 * E_{c, 6}}{E_{\text {total }}}-1\right)
\end{array}\right.
$$

where $x$ and $y$ are coordinates on the flood map $\left(256 \times 256\right.$ pixels), $E_{r, n}$ and $E_{c, n}$ are the energy signals read out by the $n$th row and $n$th column of SiPMs, respectively, and $E_{\text {total }}$ is the sum of the energy. A preliminary flood histogram was generated by tabulating the $x$ and $y$ coordinates.

The electronic noise induced by the readout circuits deterioration affects the decoding performance. A simple suppression method was utilized. The energy signals read from the $36 \mathrm{SiPMs}$ were subtracted by the preset threshold. The resultant negative signals were then set to zero:

$$
S_{\text {out }, i}=\left\{\begin{array}{cc}
S_{i n, i}-N_{t h} & \text { if } S_{i n, i}>N_{t h} \\
0 & \text { if } S_{i n, i} \leq N_{t h}
\end{array}\right.
$$

where $S_{i n, i}$ is the energy signal read from the $i$ th $\operatorname{SiPM}$ and $N_{t h}$ is the preset threshold. Flood histograms calculated using threshold values ranging from $1 \%$ to $3.5 \%$ were assessed to determine the best threshold value [44].

To quantitatively assess the performance of detector decoding, the profiles of the central column and row of the decoding spots for both the top layer and bottom layer crystals were selected for calculation of the peak-to-valley ratios. The calculation is shown in Figure 3. For each layer, the two adjacent valleys of peaks were averaged and the averaged peak-to-valley ratios were calculated.

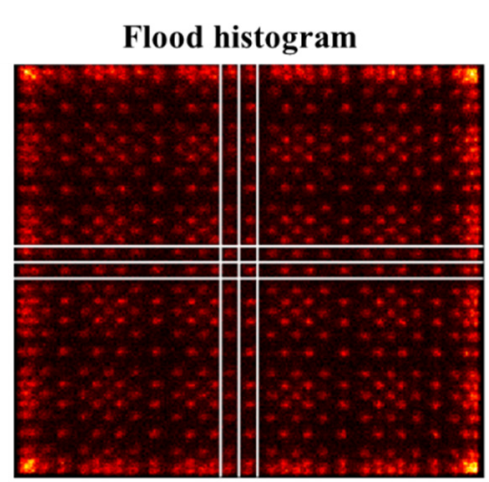

(a)

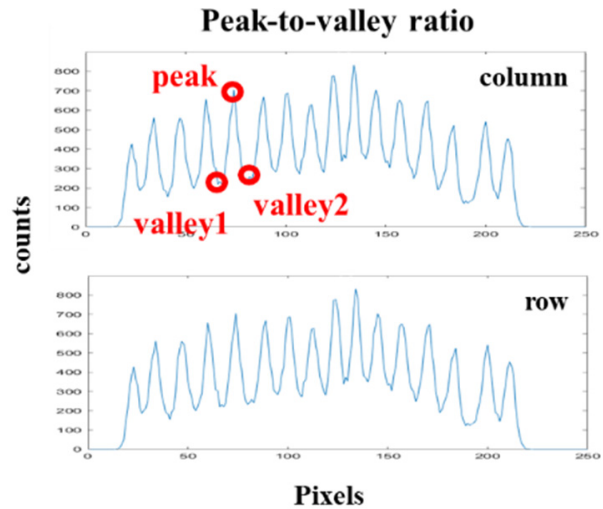

(b)

Figure 3. An illustration of the peak-to-valley ratio calculations. (a) A typical flood histogram. (b) An illustration of the calculation. The profiles of the central column and row of the spots in both the top layer and bottom layer crystals were selected for calculation of the peak-to-valley ratios.

After determining the optimal thresholds, a crystal look-up table (LUT) was generated from the flood histogram. Each decoding spot corresponds to a segmented crystal. The top layer was decoded to form a $17 \times 17$ decoding array and the bottom for an $18 \times 18$ array. The DOI can be decoded according to the locations of the $x, y$ coordinates. Then crystal energy spectra for individual crystals of both layers were extracted from the event data using the crystal LUT. The photopeak amplitudes of the energy spectra were Gaussian fitted to calculate the energy resolution. Finally, the energy spectrum and the 2D mapping of the energy resolutions of the individual crystals in the top and bottom layers were generated, respectively. The average and the standard deviation (STD) value of energy resolution were calculated. 


\section{Results}

\subsection{Decoding Results}

\subsubsection{Flood Histograms of ESR Reflectors}

The flood histograms calculated from the experimental data of the dual-layer-offset crystal array constructed with ESR reflectors are shown in Figure $4 \mathrm{a}-\mathrm{f}$. The thresholds were set from $1 \%$ to $3.5 \%$ of the photopeak with a step of $0.5 \%$. When the threshold was low $(1 \%$ and $1.5 \%)$, the flood histograms were fuzzy, and the crystal spots could not be distinguished. When the threshold was high (above $2 \%$ ), a small number of the crystal spots could be resolved. However, the decoding spots of the five crystals that were coupled with the central area of each SiPM were very intense and caused deterioration of the flood histograms. Overall, the ESR array showed poor decoding performance.

ESR Threshold $=1 \%$

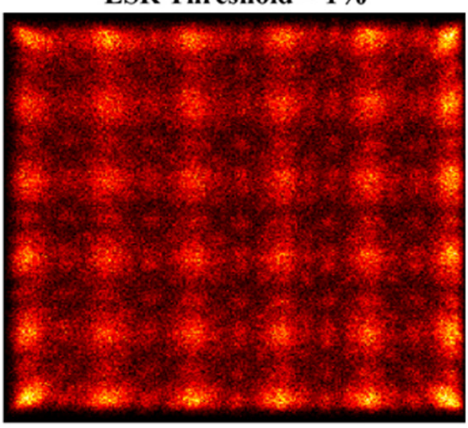

(a)

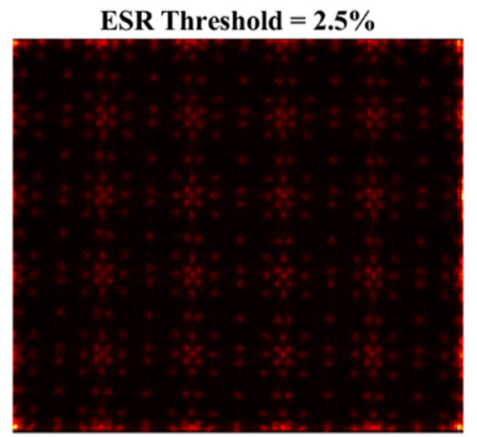

(d)
ESR Threshold $=\mathbf{1 . 5} \%$

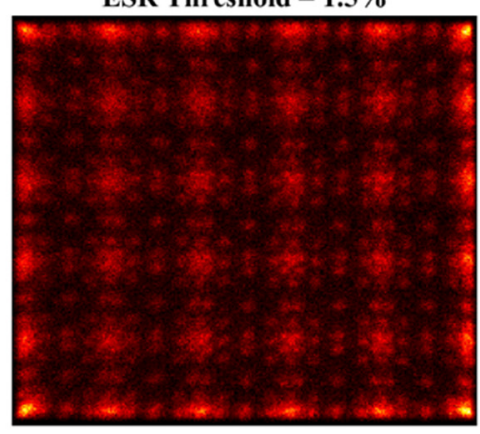

(b)

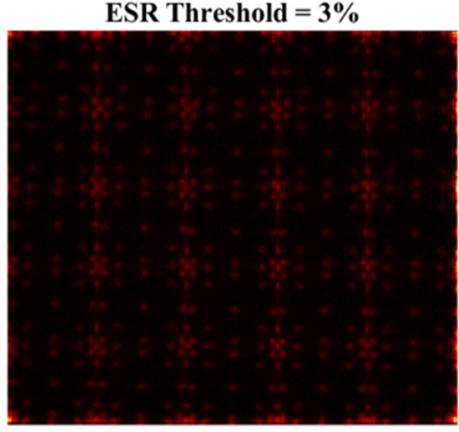

(e)

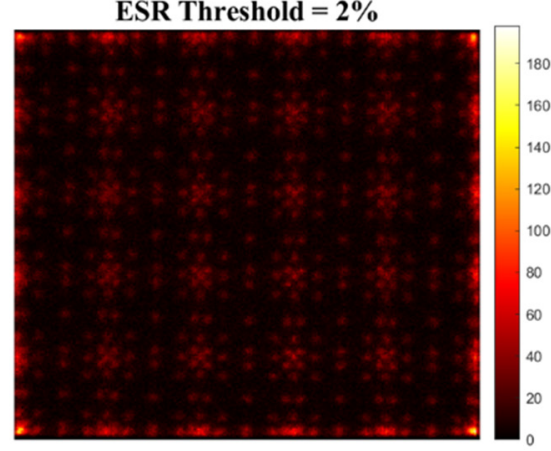

(c)

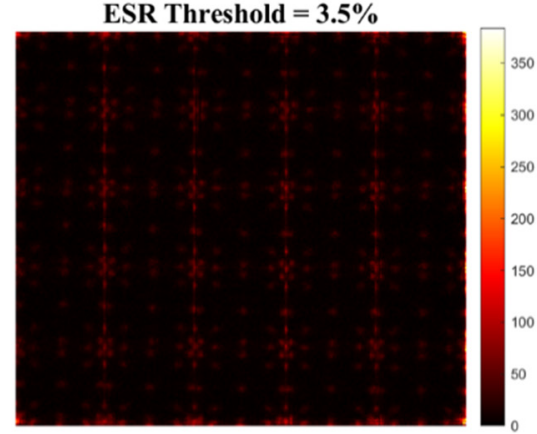

(f)

Figure 4. Flood histograms calculated from the experimental data of the dual-layer-offset crystal array constructed with ESR reflectors: (a) threshold $=1 \%$, (b) threshold $=1.5 \%$, (c) threshold $=2 \%$, (d) threshold $=2.5 \%$, (e) threshold $=3 \%$, and (f) threshold $=3.5 \%$.

Because the crystals cannot be distinguished in the crystal array constructed with ESR reflectors, the decoding spots of the crystals in the top and the bottom layers mixed. Thus, the profiles could not be precisely drawn and the peak-to-valley ratios were not calculated for the ESR reflectors.

\subsubsection{Flood Histograms of Toray Reflectors}

The flood histograms calculated from the experimental data of the dual-layer-offset crystal array constructed with Toray reflectors are shown in Figure 5a-f. The thresholds were set from $1 \%$ to $3.5 \%$ of the photopeak with a step of $0.5 \%$. The central $16 \times 16$ array of crystal spots can be distinguished unambiguously, particularly for the thresholds of $1.5 \%$ to $3 \%$ of the photopeak. However, due to edge effects, the crystal spots on the perimeter were too intense to be resolved. 


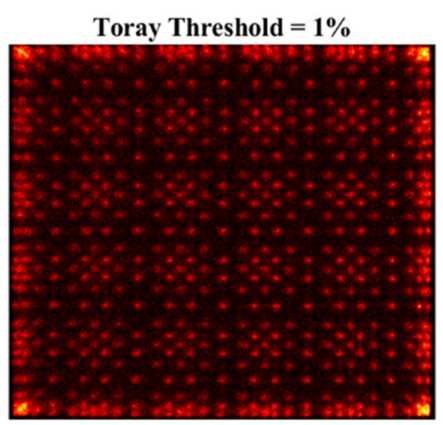

(a)

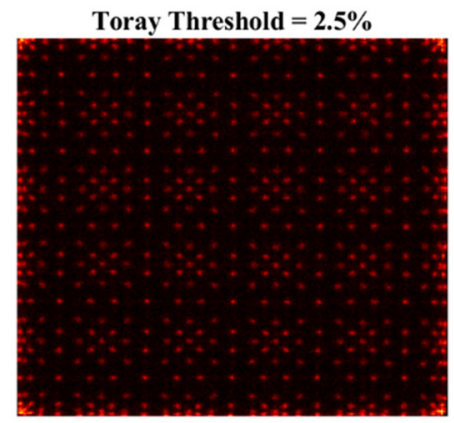

(d)
Toray Threshold $=1.5 \%$

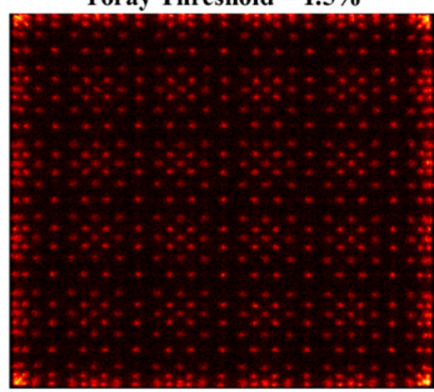

(b)

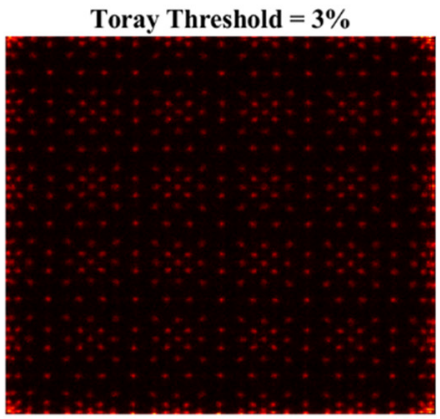

(e)

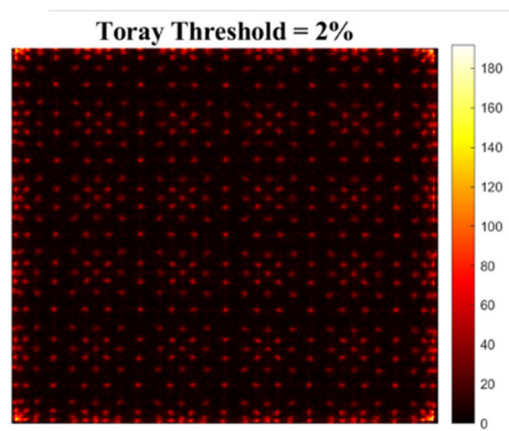

(c)

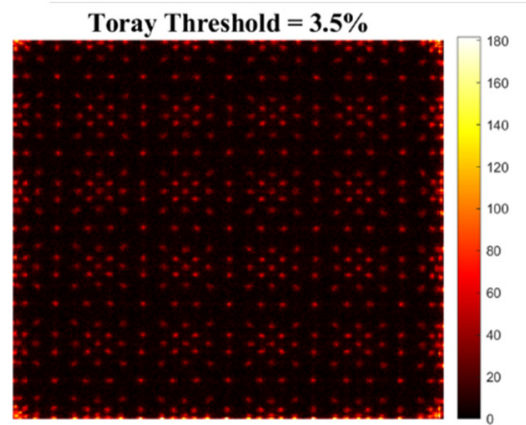

(f)

Figure 5. The flood histograms calculated from the experimental data of the dual-layer-offset crystal array constructed with Toray reflectors: (a) threshold $=1 \%$, (b) threshold $=1.5 \%$, (c) threshold $=2 \%$, (d) threshold $=2.5 \%$, (e) threshold $=3 \%$, and (f) threshold $=3.5 \%$.

To evaluate the decoding performance quantitively, the peak-to-valley ratios of the central column and the central row of the top and bottom layers were calculated. First, the crystals on the top layer and the bottom layer were classified according to the position of decoding spots, as shown in Figure 6a. Then, the relationships between the average peak-to-valley ratios and the thresholds are calculated, as shown in Figure $6 \mathrm{~b}$. The bottom crystals showed better discrimination than the top crystals. As the threshold increased from $1 \%$ to $2 \%$ of the photopeak, the average peak-to-valley ratio of the top crystals increased from 2.48 to 3.56 and the average ratio of the bottom layer increased from 3.43 to 4.73 . When the threshold was set higher than $2 \%$, the peak-to-valley ratio remained almost constant for both layers. Based on our previous experience [44], the optimal threshold was $2 \%$, because higher thresholds cause distortion of the signal and decoding specks. The average peak-to-valley ratios at a threshold value of $2 \%$ for top and bottom crystals were 3.56 and 4.73 , respectively. 


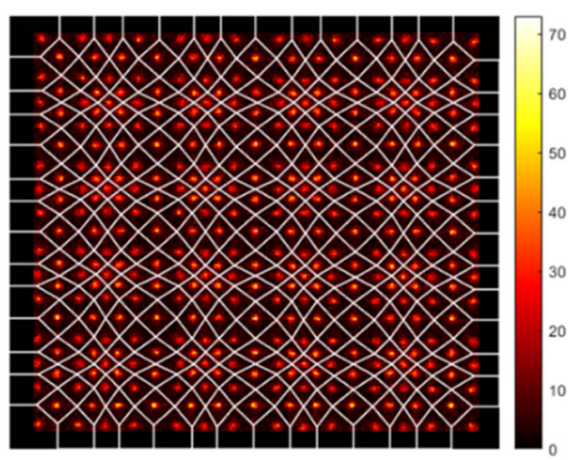

(a)

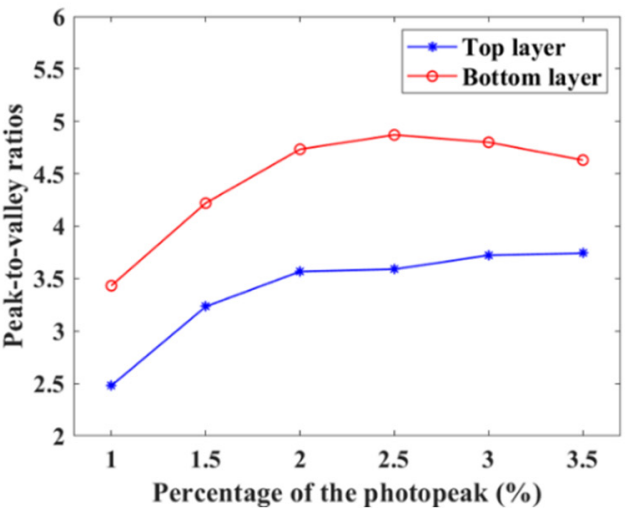

(b)

Figure 6. (a) Classification of the spots for the top and bottom crystals in the flood histogram of the Toray array with a threshold of $2 \%$ of the photopeak. (b) Plot of the peak-to-valley ratios versus the threshold (percentage of the photopeak).

\subsubsection{Flood Histograms of $\mathrm{BaSO}_{4}$ Reflectors}

The flood histograms calculated from the experimental data of the dual-layer-offset crystal array constructed with $\mathrm{BaSO}_{4}$ reflectors are shown in Figure $7 \mathrm{a}-\mathrm{f}$. As the threshold value ranged from $1 \%$ to $3.5 \%$ of the photopeak, the flood histogram showed subtle differences, mainly located on the periphery. Nevertheless, almost all of the crystal spots, both the $17 \times 17$ crystal array in the top layer and the $18 \times 18$ crystal array in the bottom layer, are bright enough to be unambiguously distinguished, even those on the periphery. Overall, the flood histograms of $\mathrm{BaSO}_{4}$ array are the best of all the arrays evaluated.

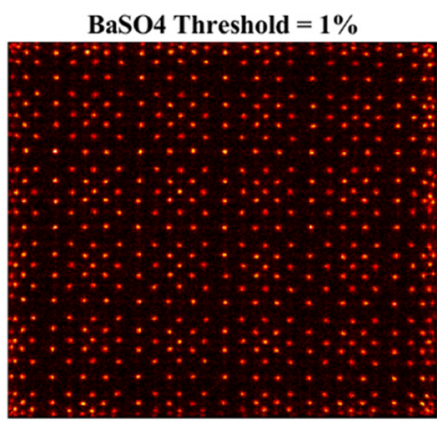

(a)

BaSO4 Threshold $=\mathbf{2 . 5} \%$

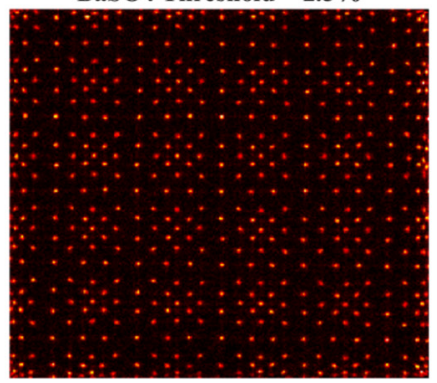

(d)

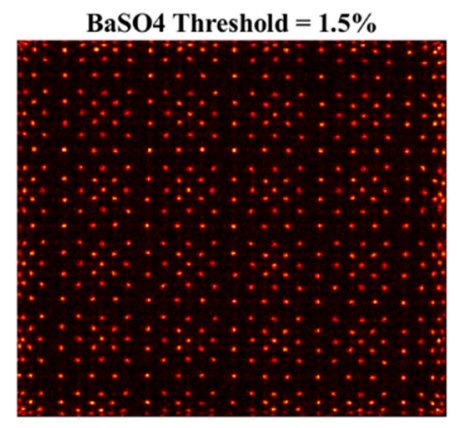

(b)

BaSO4 Threshold $=3 \%$

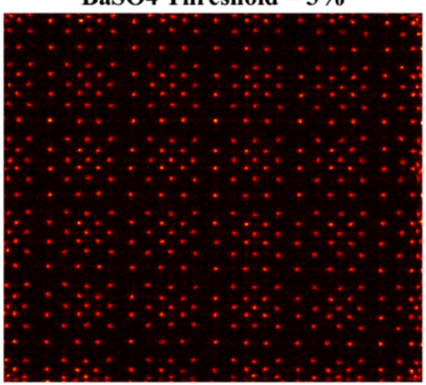

(e)

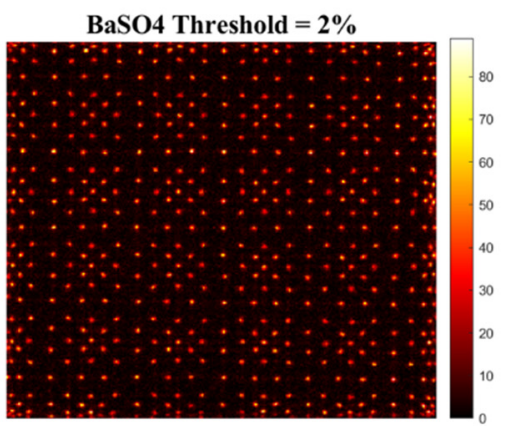

(c)

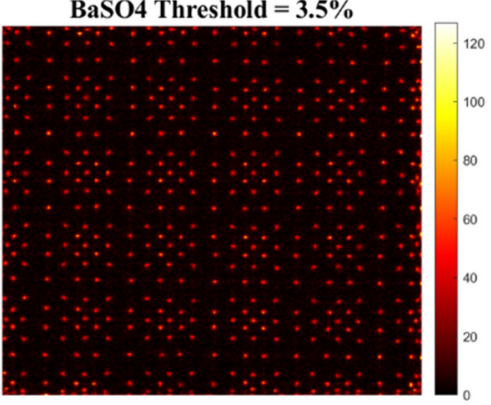

(f)

Figure 7. Flood histograms calculated from the experimental data of the dual-layer-offset crystal array constructed with $\mathrm{BaSO}_{4}$ reflectors: (a) threshold $=1 \%$, (b) threshold $=1.5 \%$, (c) threshold $=2 \%$, (d) threshold $=2.5 \%$, (e) threshold $=3 \%$, and (f) threshold $=3.5 \%$. 
An analysis of the peak-to-valley ratios of the central column and the central row of the top and bottom layers was conducted for the $\mathrm{BaSO}_{4}$ array. The crystals on the top and bottom layers were classified and the relation between the average peak-to-valley ratios and the threshold values were calculated, as shown in Figure 8a,b. As with the Toray array, the bottom crystals were more easily distinguished than the top crystals. As the threshold value was increased from $1 \%$ to $2.5 \%$ of the photopeak, the average peak-to-valley ratio of the top layer of crystals increased from 5.53 to 6.18 and the average ratio of the bottom layer increased from 6.85 to 7.50 . The maximum peak-to-valley ratios for the top and bottom crystals were 6.18 and 7.50 , respectively, at a threshold of $2.5 \%$ of the photopeak. The peak-to-valley ratios were approximately twice as high as those of the Toray array. It can therefore be concluded that the $\mathrm{BaSO}_{4}$ reflectors have an enormous advantage over Toray reflectors in energy resolution when assembling crystal arrays.

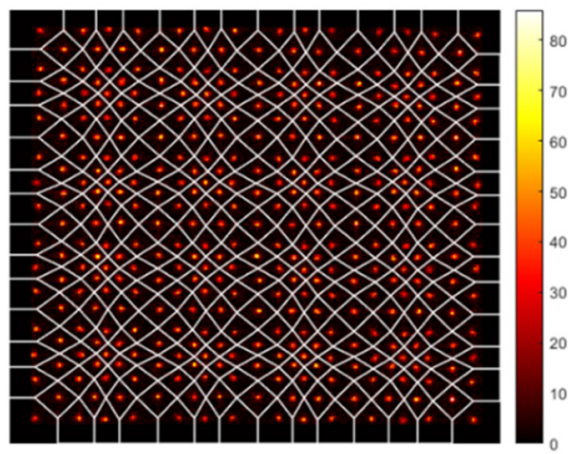

(a)

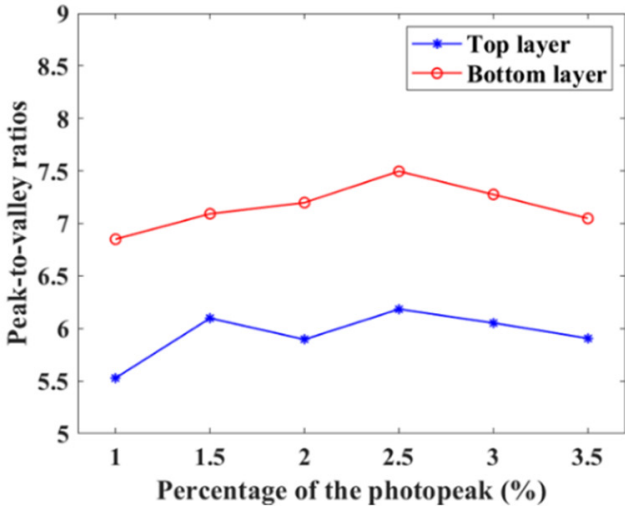

(b)

Figure 8. (a) Classification of the spots for the top and bottom crystals in the flood histogram of the $\mathrm{BaSO}_{4}$ array with a threshold value of $2.5 \%$ of the photopeak. (b) Plot of the peak-to-valley ratios versus the threshold value (percentage of the photopeak).

\subsection{Energy Resolution}

\subsubsection{Energy Resolution of ESR Reflectors}

The energy spectrum of the crystal array constructed with ESR reflectors is shown in Figure 9. The energy resolution is $19.18 \%$. Note that because the flood histogram of ESR array cannot be resolved, the individual energy resolution cannot be calculated.

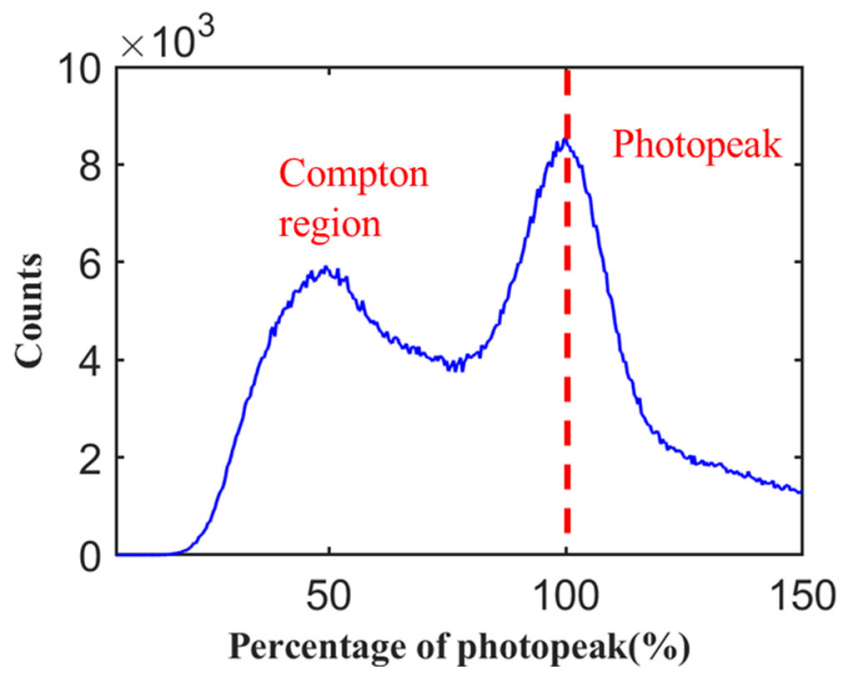

Figure 9. The energy spectrum of the crystal array constructed with ESR reflectors. 


\subsubsection{Energy Resolution of Toray Reflectors}

Based on the flood histograms in Section 3.1.2, the central $13 \times 13$ crystal array of the top crystal layer and the central $14 \times 14$ array of the bottom crystal layer were easily distinguished. This allowed the energy resolutions of the crystal strips of the dual-layeroffset crystal array constructed with Toray reflectors to be calculated, as shown in Figure 10; a 2D mapping and a histogram of the energy resolutions of the individual 169 crystals of the top layer and the 196 crystals of the bottom layer, respectively, were generated. The average value and SD of the energy resolution of top and bottom crystals were $19.76 \% \pm 1.95 \%$, and $20.52 \% \pm 2.13 \%$, respectively. The energy performance of the top layer was approximately equal to that of the bottom layer.

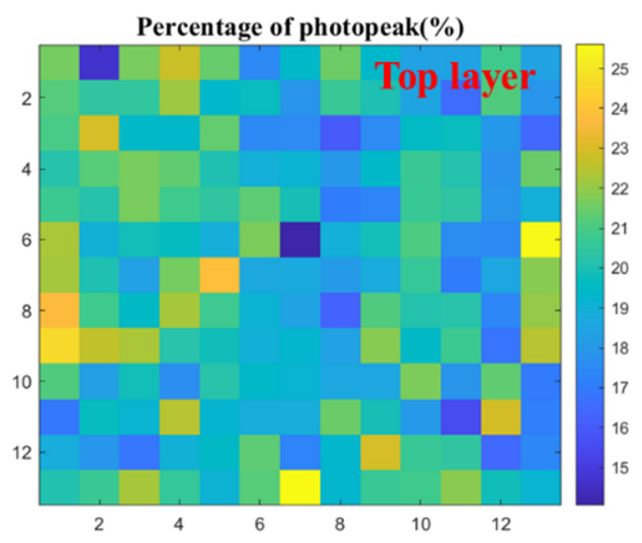

(a)

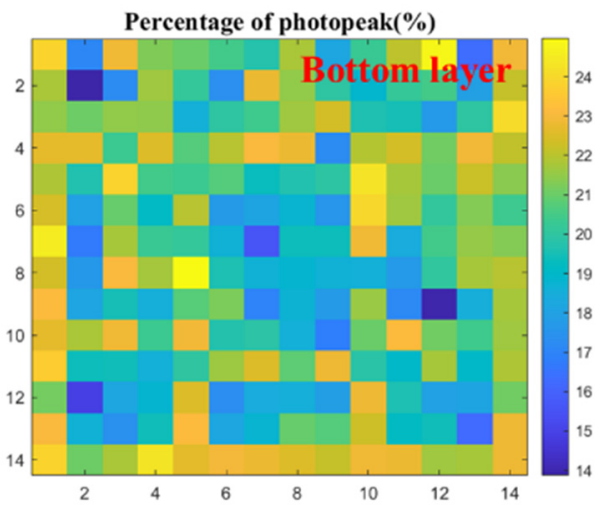

(c)

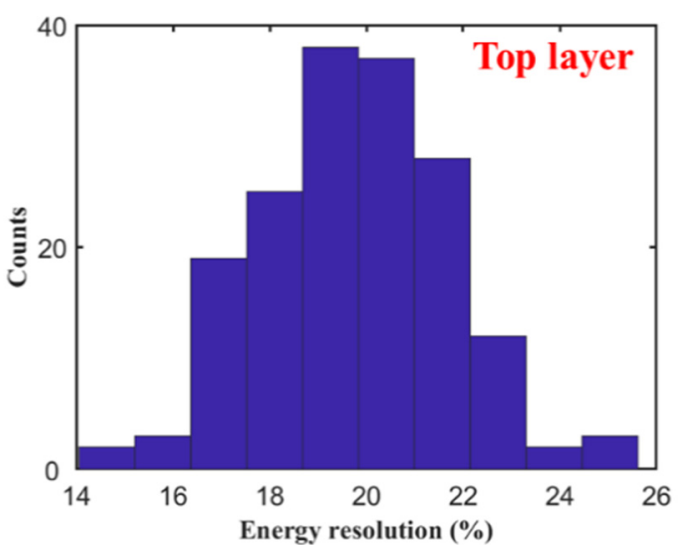

(b)

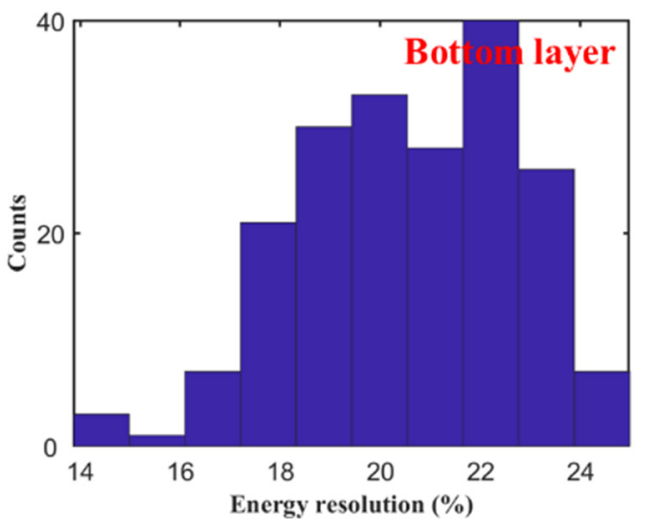

(d)

Figure 10. 2D mappings of the energy resolutions of the Toray array: (a) the 169 individual crystals of the $13 \times 13$ crystal array in the top layer and (c) the 196 individual crystals of the $14 \times 14$ crystal array in the bottom layer. The histogram of the energy resolutions of (b) 169 individual crystals of the $13 \times 13$ crystal array in the top layer and (d) the 196 individual crystals of the $14 \times 14$ crystal array in the bottom layer.

\subsubsection{Energy Resolutions of $\mathrm{BaSO}_{4}$ Reflectors}

Based on the flood histograms in Section 3.1.3, the central $13 \times 13$ crystal array of the top layer and the central $14 \times 14$ array of the bottom layer were easily distinguished. This allowed the energy resolutions of the crystal strips of the dual-layer-offset crystal array constructed with $\mathrm{BaSO}_{4}$ reflectors to be calculated, as shown in Figure 11. A 2D mapping and histogram of the energy resolutions of the individual 169 crystals of the top layer and the 196 crystals of the bottom layer, respectively, were generated. The average and the SD of the energy resolutions of the top and bottom crystals were $11.07 \% \pm 1.01 \%$, 
and $11.46 \% \pm 1.35 \%$, respectively. As in the Toray array, the energy performance of the top layer was approximately equal to that of the bottom layer. The $\mathrm{BaSO}_{4}$ reflectors have an enormous advantage over the Toray reflectors in energy resolution when assembling crystal arrays.

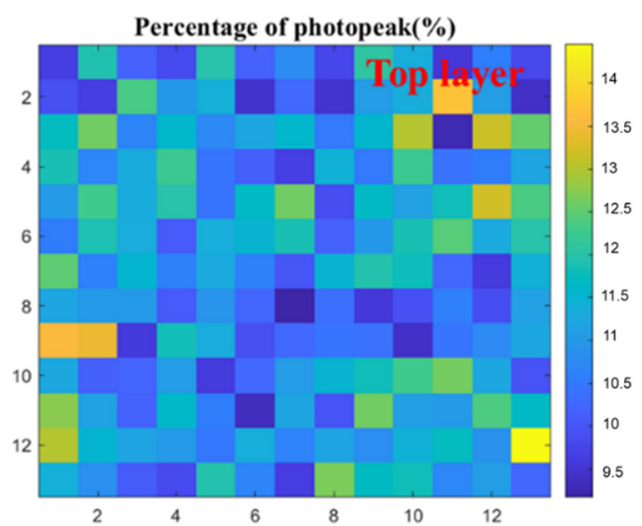

(a)

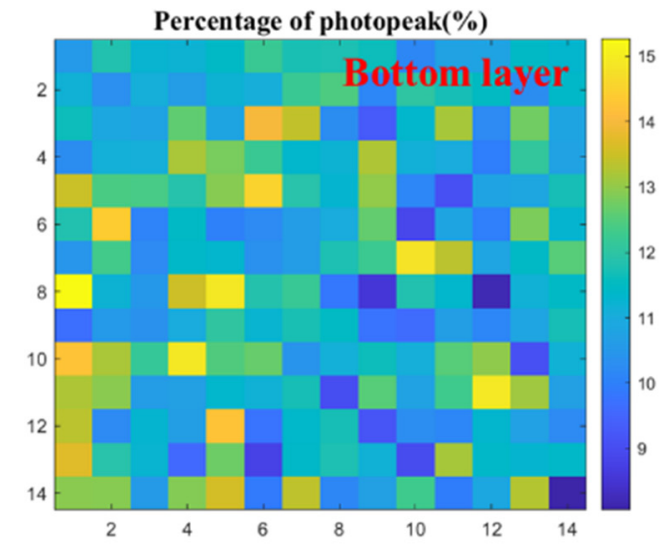

(c)

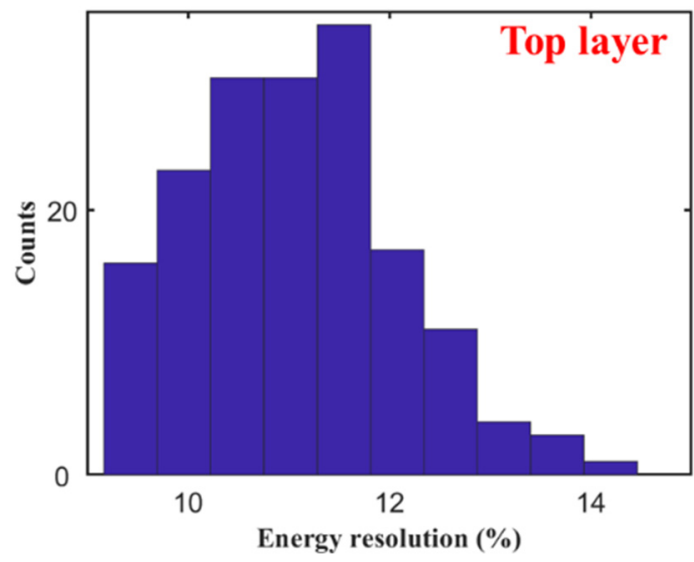

(b)

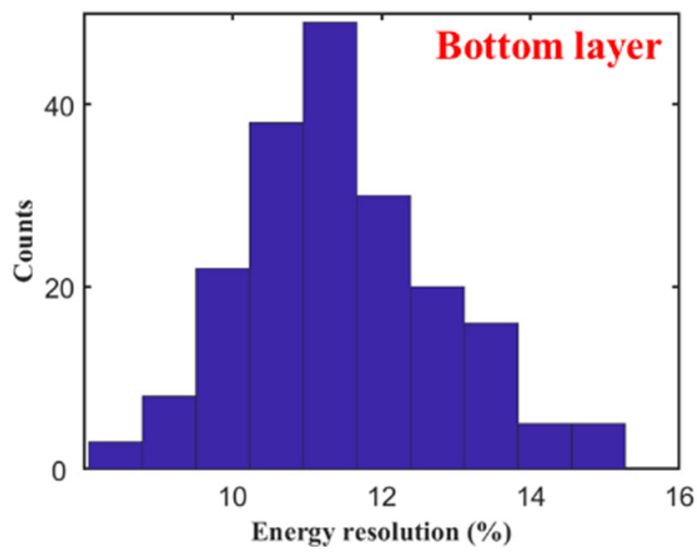

(d)

Figure 11. 2D mapping of the energy resolutions of the $\mathrm{BaSO}_{4}$ array: (a) the 169 individual crystals of the $13 \times 13$ crystal array in the top layer and (c) the 196 individual crystals of the $14 \times 14$ crystal array in the bottom layer. The histogram of the energy resolutions of (b) the 169 individual crystals of the $13 \times 13$ crystal array in the top layer and (d) the 196 individual crystals of the $14 \times 14$ crystal array in the bottom layer.

\subsection{Coincidence Time Resolution}

A high-performance test system with good timing resolution was used in this study [6]. The time resolutions of the three dual-layer-offset arrays with different reflectors were tested and the results are shown in Figure 12. The CTRs for $\mathrm{BaSO}_{4}$, Toray film, and ESR reflectors are 386.08 ps, 402.34 ps, and 423.56 ps, respectively. 


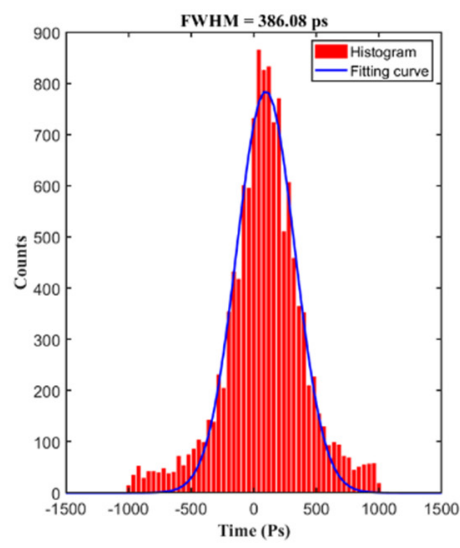

(a)

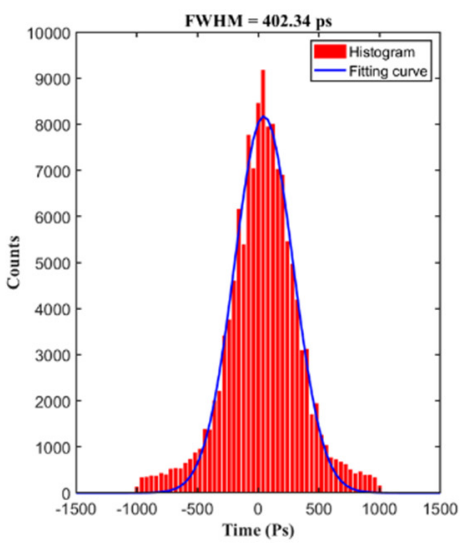

(b)

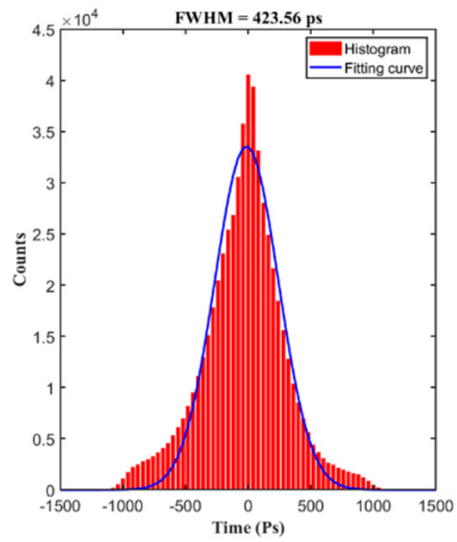

(c)

Figure 12. The coincident time resolutions of the crystal arrays with (a) $\mathrm{BaSO}_{4}$ reflectors, (b) Toray film reflectors (c) ESR reflectors.

\section{Discussion and Conclusions}

Three dual-layer-offset LYSO detectors with different reflectors (ESR, Toray, and $\mathrm{BaSO}_{4}$ ) were developed for a high-resolution PET system. The top layer consisted of a $17 \times 17$ array of crystals $1 \times 1 \times 6.5 \mathrm{~mm}^{3}$ in size and the bottom layer consisted of an $18 \times 18$ array of crystals $1 \times 1 \times 9.5 \mathrm{~mm}^{3}$ in size. Because the bottom layer has a lower stop efficiency, the bottom crystal was designed to be longer than the top layer. An optimized interactiondecoding algorithm using the COG and threshold methods was applied to generate flood histograms. The peak-to-valley ratios of the decoding spots and the energy resolution of each individual crystal were calculated to evaluate the detector performance.

The electronic noise from the SiPMs can severely deteriorate the crystal flood histograms when the COG method is used to decode the interaction. One noise-suppression method was utilized in this study: the 36 channels of energy signals were subtracted by a preset threshold, and the negative signals set to zero. The decoding performance is significantly improved while the energy spectra before and after the noise suppression exhibit negligible differences. The array constructed with ESR reflectors could not be clearly resolved even with a wide range of threshold values, while the other two arrays were unambiguously distinguished. The optimal threshold values for the arrays constructed with Toray and $\mathrm{BaSO}_{4}$ reflectors are $2 \%$ and $2.5 \%$ of the photopeak, respectively. The peakto-valley ratios obtained from the $\mathrm{BaSO}_{4}$ reflectors were approximately twice as high as the results from the Toray reflectors. It can therefore be concluded that the $\mathrm{BaSO}_{4}$ reflectors have an enormous advantage over Toray reflectors in energy resolution when assembling crystal arrays. In addition, the crystals of the top layer had lower peak-to-valley ratios than those of bottom layer in both arrays.

Individual elements in the flood map of the detector with ESR reflectors could not be identified clearly. Photon spreading across the SiPMs is especially important for specular reflectors such as the ESR where the light spread is limited, compared to diffused reflectors such as $\mathrm{BaSO}_{4}$. In our study, neither light guides nor optical glue were used between the two layers of crystals and no light guide was used between the crystal arrays and SiPM board. Therefore, the positioning of the ESR reflector array was poor. $\mathrm{BaSO}_{4}$ is slightly transparent to light and, thus, the spread range of scintillating photons is wide in the crystal array with $\mathrm{BaSO}_{4}$ reflectors.

The energy resolutions of the central $13 \times 13$ crystal array of the top layer and the central $14 \times 14$ array of the bottom layer were calculated. The average and the STD of the energy resolutions of the top and bottom crystals for the Toray array were $19.76 \% \pm 1.95 \%$ and $20.52 \% \pm 2.13 \%$, respectively, while for the $\mathrm{BaSO}_{4}$ array they were $11.07 \% \pm 1.01 \%$ and $11.46 \% \pm 1.35 \%$, respectively. The energy performance of the Toray array was markedly inferior to that of the $\mathrm{BaSO}_{4}$ array. In addition, although the bottom crystals were directly 
coupled to the SiPMs, the energy resolutions of the top crystals were slightly better. This is because half of the scintillating photons generated from the interactions that occur in the bottom layer radiate upward and enter the top layer, which greatly lengthens the photon path.

In summary, the dual-layer-offset PET detector constructed with $\mathrm{BaSO}_{4}$ reflectors performed much better than the other two reflectors in crystal identification and energy resolution. The average peak-to-valley ratio and the energy resolution were approximately 7 and 11\%, respectively. This study provides guidance for choosing scintillator reflectors for multi-layer dedicated DOI detectors designed for small-animal PET imaging.

Author Contributions: Conceptualization: X.Z. and S.X.; methodology: X.Z.; formal analysis: X.Y. and Z.Z.; investigation: H.Y.; data curation: H.Z. and Y.Z.; writing—original draft preparation: X.Z. and S.X.; writing - review and editing: Z.G. and Q.P.; funding acquisition, Q.P. and J.X. All authors have read and agreed to the published version of the manuscript.

Funding: This work was supported by the National Natural Science Foundation of China (51627807), the Shenzhen Science and Technology Program (RCBS20200714114920148), the Guangdong Basic and Applied Basic Research Foundation (2020A1515110588), and Shenzhen Science and Technology Program (KQTD2017033015530192).

Institutional Review Board Statement: Not applicable.

Informed Consent Statement: Not applicable.

Data Availability Statement: Not applicable.

Acknowledgments: We sincerely thank the Micro and Nano Fabrication and Measurement Laboratory of Mechanical Science and Engineering in HUST.

Conflicts of Interest: The authors declare no conflict of interest.

\section{References}

1. Stickel, J.R.; Cherry, S.R. High-resolution PET detector design: Modelling components of intrinsic spatial resolution. Phys. Med. Biol. 2005, 50, 179-195. [CrossRef]

2. Cherry, S.R. In vivo molecular and genomic imaging: New challenges for imaging physics. Phys. Med. Biol. 2004, 49, R13-R48. [CrossRef]

3. Myers, R. The biological application of small animal PET imaging. Nucl. Med. Biol. 2001, 28, 585-593. [CrossRef]

4. Townsend, D.W. Multimodality imaging of structure and function. Phys. Med. Biol. 2008, 53, R1-R39. [CrossRef] [PubMed]

5. Eriksson, L.; Townsend, D.; Conti, M.; Eriksson, M.; Rothfuss, H.; Schmand, M.; Casey, M.E.; Bendriem, B. An investigation of sensitivity limits in PET scanners. Nucl. Instrum. Methods Phys. Res. Sect. A 2007, 580, 836-842. [CrossRef]

6. Xie, S.; Zhang, X.; Zhang, Y.; Ying, G.; Huang, Q.; Xu, J.; Peng, Q. Evaluation of Various Scintillator Materials in Radiation Detector Design for Positron Emission Tomography (PET). Crystals 2020, 10, 869. [CrossRef]

7. Moses, W.W. Fundamental limits of spatial resolution in PET. Nucl. Instrum. Methods Phys. Res. Sect. A 2011, 648, S236-S240. [CrossRef]

8. Behnamian, H.; Yousefnejad, S.; Shafiee, M.; Rafiei, A. Study of two-layer tapered depth of interaction PET detector. Appl. Radiat. Isot. 2021, 174, 109731. [CrossRef] [PubMed]

9. Choghadi, M.A.; Huang, S.C.; Shimazoe, K.; Takahashi, H. Evaluation of dual-ended readout GAGG-based DOI-PET detectors with different surface treatments. Med. Phys. 2021, 48, 3470-3478. [CrossRef]

10. Kang, H.G.; Yamaya, T.; Han, Y.B.; Song, S.H.; Ko, G.B.; Lee, J.S.; Hong, S.J. Crystal surface and reflector optimization for the SiPM-based dual-ended readout TOF-DOI PET detector. Biomed. Phys. Eng. Express 2020, 6. [CrossRef]

11. Kuang, Z.; Wang, X.; Ren, N.; Wu, S.; Zhang, M.; Gao, J.; Sang, Z.; Hu, Z.; Du, J.; Yang, Y. Progress of a MRI compatible small animal PET scanner using dual-ended readout detectors. J. Nucl. Med. 2019, 60, 527.

12. Wang, Y.; Seidel, J.; Tsui, B.M.; Vaquero, J.J.; Pomper, M.G. Performance evaluation of the GE healthcare eXplore VISTA dual-ring small-animal PET scanner. J. Nucl. Med. 2006, 47, 1891-1900. [PubMed]

13. Kitamura, K.; Mizuta, T.; Iwata, H. Development of "Clairvivo PET" small animal PET scanner. Shimadzu Hyoron 2008, 64, 107-115.

14. Pourashraf, S.; Cates, J.W.; Lee, M.S.; Levin, C.S. Pulse Shape Discrimination and Energy Measurement in Phoswich Detectors Using Gated-Integrator Circuit. In Proceedings of the IEEE Nuclear Science Symposium and Medical Imaging Conference (NSS/MIC), Manchester, UK, 10 October-2 November 2019; pp. 1-2.

15. Dahlbom, M.; MacDonald, L.; Eriksson, L.; Paulus, M.; Andreaco, M.; Casey, M.; Moyers, C. Performance of a YSO/LSO phoswich detector for use in a PET/SPECT system. IEEE Trans. Nucl. Sci. 1997, 44, 1114-1119. [CrossRef] 
16. Tyagi, M.; Rawat, S.; Kumar, G.A.; Gadkari, S.C. A novel versatile phoswich detector consisting of single crystal scintillators. Nucl. Instrum. Methods A 2020, 951, 162982. [CrossRef]

17. Wei, Q.; Ma, T.; Jiang, N.; Xu, T.; Lyu, Z.; Hu, Y.; Liu, Y. A side-by-side LYSO/GAGG phoswich detector aiming for SPECT imaging. Nucl. Instrum. Methods A 2020, 953, 163242. [CrossRef]

18. Xu, J.; Liu, J.; Chen, X. A well typed phoswich detector consisting of CsI and plastic scintillators for low level radioactivity measurements. Appl. Radiat. Isot. 2021, 169, 109462. [CrossRef]

19. Zorloni, G.; Cova, F.; Caresana, M.; Benedetto, M.D.; Hostaša, J.; Fasoli, M.; Villa, I.; Veronese, I.; Fazzi, A.; Vedda, A. Neutron/ $\gamma$ discrimination by an emission-based phoswich approach. Radiat. Meas. 2019, 129, 106203. [CrossRef]

20. Eriksson, L.; Melcher, C.L.; Eriksson, M.; Rothfuss, H.; Grazioso, R.; Aykac, M. Design Considerations of Phoswich Detectors for High Resolution Positron Emission Tomography. IEEE Trans. Nucl. Sci. 2009, 56, 182-188. [CrossRef]

21. Lee, M.S.; Cates, J.W.; Gonzalez-Montoro, A.; Levin, C.S. High-resolution time-of-flight PET detector with 100 ps coincidence time resolution using a side-coupled phoswich configuration. Phys. Med. Biol. 2021, 66, 125007. [CrossRef]

22. Min, S.; Seo, B.; Roh, C.; Hong, S.; Cheong, J. Phoswich Detectors in Sensing Applications. Sensors 2021, 21, 4047. [CrossRef] [PubMed]

23. Wilkinson, D.H. The Phoswich-A Multiple Phosphor. Rev. Sci. Instrum. 1952, 23, 414-417. [CrossRef]

24. Prout, D.L.; Gu, Z.; Shustef, M.; Chatziioannou, A.F. A digital phoswich detector using time-over-threshold for depth of interaction in PET. Phys. Med. Biol. 2020, 65, 245017. [CrossRef] [PubMed]

25. Wienhard, K.; Schmand, M.; Casey, M.E.; Baker, K.; Bao, J.; Eriksson, L.; Jones, W.F.; Knoess, C.; Lenox, M.; Lercher, M. The ECAT HRRT: Performance and first clinical application of the new high resolution research tomograph. IEEE Trans. Nucl. Sci. 2002, 49, 104-110. [CrossRef]

26. Son, J.; Lee, M.S.; Lee, J.S. A depth-of-interaction PET detector using a stair-shaped reflector arrangement and a single-ended scintillation light readout. Phys. Med. Biol. 2017, 62, 465-483. [CrossRef]

27. Zhao, B.; Kuang, Z.; Sun, M.; Zhang, C.; Wang, X.; Sang, Z.; Yang, Q.; Wu, S.; Gao, J.; Ren, N.; et al. Depth encoding PET detectors using single layer crystal array with different reflector arrangements along depths. Nucl. Instrum. Methods A 2019, 945, 162600. [CrossRef]

28. Zhang, X.; Xie, S.; Yang, J.; Weng, F.; Xu, J.; Huang, Q.; Peng, Q. A depth encoding PET detector using four-crystals-to-one-SiPM coupling and light-sharing window method. Med. Phys. 2019, 46, 3385-3398. [CrossRef]

29. LaBella, A.; Cao, X.; Petersen, E.; Lubinsky, R.; Biegon, A.; Zhao, W.; Goldan, A.H. High-Resolution Depth-Encoding PET Detector Module with Prismatoid Light-Guide Array. J. Nucl. Med. 2020, 61, 1528-1533. [CrossRef]

30. LaBella, A.; Cao, X.; Zeng, X.; Cao, X.; Zeng, X. Sub-2 mm depth of interaction localization in PET detectors with prismatoid light guide arrays and single-ended readout using convolutional neural networks. Med. Phys. 2021, 48, 1019-1025. [CrossRef] [PubMed]

31. Balcerzyk, M.; Kontaxakis, G.; Delgado, M.; Garcia-Garcia, L.; Correcher, C.; Gonzalez, A.J.; Gonzalez, A.; Rubio, J.L.; Benlloch, J.M.; Pozo, M.A. Initial performance evaluation of a high resolution Albira small animal positron emission tomography scanner with monolithic crystals and depth-of-interaction encoding from a user's perspective. Meas. Sci. Technol. 2009, $20,104011$. [CrossRef]

32. Thompson, C.; Stortz, G.; Goertzen, A.; Berg, E.; Retière, F.; Kozlowski, P.; Ryner, L.; Sossi, V.; Zhang, X. Comparison of single and dual layer detector blocks for pre-clinical MRI-PET. Nucl. Instrum. Methods A 2013, 702, 56-58. [CrossRef]

33. Seidel, J.; Vaquero, J.J.; Siegel, S.; Gandler, W.R.; Green, M.T. Depth Identification Accuracy of a Three Layer Phoswicfi PET Detector Module. IEEE Trans. Nucl. Sci. 1999, 46, 485-490. [CrossRef]

34. Hong, S.J.; Kwon, S.I.; Ito, M.; Lee, G.S.; Sim, K.-S.; Park, K.S.; Rhee, J.T.; Lee, J.S. Concept Verification of Three-Layer DOI Detectors for Small Animal PET. IEEE Trans. Nucl. Sci. 2008, 55, 912-917. [CrossRef]

35. Wei, Q.; Ma, T.; Xu, T.; Zeng, M.; Gu, Y.; Dai, T.; Liu, Y. Crystal identification for a dual-layer-offset LYSO based PET system via Lu-176 background radiation and mean shift algorithm. Phys. Med. Biol. 2018, 63, 02NT01. [CrossRef] [PubMed]

36. Zhang, X.; Stortz, G.; Sossi, V.; Thompson, C.J.; Retiere, F.; Kozlowski, P.; Thiessen, J.D.; Goertzen, A.L. Development and evaluation of a LOR-based image reconstruction with 3D system response modeling for a PET insert with dual-layer offset crystal design. Phys. Med. Biol. 2013, 58, 8379-8399. [CrossRef]

37. Ito, M.; Lee, J.S.; Kwon, S.I.; Lee, G.S.; Hong, B.; Lee, K.S.; Sim, K.-S.; Lee, S.J.; Rhee, J.T.; Hong, S.J. A_Four-Layer DOI Detector With a Relative Offset for Use in an Animal PET System. IEEE Trans. Nucl. Sci. 2010, 57, 976-981. [CrossRef]

38. Chung, Y.H.; Hwang, J.Y.; Baek, C.-H.; Lee, S.-J.; Ito, M.; Lee, J.S.; Hong, S.J. Monte Carlo simulation of a four-layer DOI detector with relative offset in animal PET. Nucl. Instrum. Methods A 2011, 626-627, 43-50. [CrossRef]

39. Kuang, Z.; Wang, X.; Li, C.; Deng, X.; Feng, K.; Hu, Z.; Fu, X.; Ren, N.; Zhang, X.; Zheng, Y.; et al. Performance of a high-resolution depth encoding PET detector using barium sulfate reflector. Phys. Med. Biol. 2017, 62, 5945-5958. [CrossRef]

40. Du, J.; Wang, Q.; Liu, C.; Qi, J.; Cherry, S.R. Performance evaluation of dual-ended readout PET detectors based on BGO arrays with different reflector arrangements. Phys. Med. Biol. 2021, 66, 215001. [CrossRef] [PubMed]

41. Ren, S.; Yang, Y.; Cherry, S.R. Effects of reflector and crystal surface on the performance. Med. Phys. 2014, 41. [CrossRef]

42. Kang, H.G.; Nishikido, F.; Yamaya, T. A staggered 3-layer DOI PET detector using $\mathrm{BaSO}_{4}$ reflector for enhanced crystal identification and inter-crystal scattering event discrimination capability. Biomed. Phys. Eng. Express 2021, 7, 035018. [CrossRef] [PubMed] 
43. Zhao, Z.; Xie, S.; Zhang, X.; Yang, J.; Huang, Q.; Xu, J.; Peng, Q. An advanced 100-channel readout system for nuclear imaging. IEEE Trans. Instrum. Meas. 2019, 68, 3200-3210. [CrossRef] [PubMed]

44. Zhang, X.; Yu, H.; Xie, Q.; Xie, S.; Ye, B.; Guo, M.; Zhao, Z.; Huang, Q.; Xu, J.; Peng, Q. Design study of a PET detector with $0.5 \mathrm{~mm}$ crystal pitch for high-resolution preclinical imaging. Phys. Med. Biol. 2021, 66, 135013. [CrossRef] [PubMed] 